\title{
A Novel Formulation of Economic Model Predictive Control for Periodic Operations
}

\author{
Ye Wang ${ }^{1}$, David Muñoz de la Peña ${ }^{2}$, Vicenç Puig ${ }^{1}$ and Gabriela Cembrano ${ }^{1,3}$
}

\begin{abstract}
This paper proposes a novel formulation of economic model predictive control (MPC) for linear systems with periodic operations. In this economic MPC design, the optimal periodic trajectory from an economic point of view is unknown, hence it is not possible to follow a standard control strategy in which the MPC uses this trajectory to define a terminal constraint to guarantee closed-loop convergence. The economic cost function is optimized with a periodicity constraint at each time step considering all periodic trajectories in a period including the current state. The recursive feasibility and closed-loop convergence to the optimal periodic trajectory are analyzed using the Karush-Kuhn-Tucker conditions. Finally, two simulations are provided to demonstrate the main results.
\end{abstract}

\section{INTRODUCTION}

During the past decade, economic model predictive control (MPC) has attracted an increasing attention [1], [2]. Instead of regulating the system to a given reference minimizing a cost function based on the state and input deviation from the reference, economic MPC aims to optimize an economic performance index. In this case, economic cost functions are not necessarily quadratic or positive definite with respect to the given trajectories or references. From the application point of view, economic MPC is a suitable and useful control strategy that has been applied to a variety of industrial applications, such as water distribution networks [3], [4], wastewater treatment processes [5], smart grids [6], [7], and chemical processes [8], [9].

Among these applications, periodic system behavior arises in some specific cases, such as water distribution networks [10] and electrical networks [6]. As shown in [11], [12], [13], the operational management of a water distribution network can be realized to obtain a periodic optimal trajectory of storage tanks taking into account the daily water demands and time-varying electricity price. Hence, the periodic behavior can be integrated into the controller design.

In terms of economic MPC, the closed-loop stability is an open challenge that has currently being researched. An comprehensive review on economic MPC is presented in [14] and the closed-loop stability and performance are

\footnotetext{
${ }^{1}$ Y. Wang, V. Puig and G. Cembrano are with Advanced Control Systems (SAC) Research Group at Institut de Robòtica i Informàtica Industrial (IRI), CSIC-UPC, Universitat Politècnica de Catalunya-BarcelonaTech (UPC), C/. Llorens i Artigas 4-6. 08028 Barcelona, Spain. E-mail: ywangeiri.upc.edu, vicenc.puig@upc.edu, cembranodiri.upc.edu

${ }^{2}$ D. Muñoz de la Peña is with Departamento de Ingeniería de Sistemas y Automática, Escuela Superior de Ingenieros, Universidad de Sevilla, Avenida de los Descubrimientos, S/N, 41092 Sevilla, Spain. E-mail: dmunoz@us.es

${ }^{3}$ G. Cembrano is also with CETaqua, Water Technology Centre, Ctra. d'Esplugues 75, Cornellà de Llobregat, 08940 Barcelona, Spain.
}

also addressed. In [15], [16], [17], stability analysis of economic MPC is established under the strong duality or the dissipativity assumptions. Within these strategies, the optimal steady trajectories are first given and set in the MPC loop by terminal cost or constraint. In [18], economic MPC with extended prediction horizon is designed based on an auxiliary controller. An additional term with the auxiliary control law is included in the cost function in order to guarantee closed-loop convergence. Besides, economic MPC without terminal constraints is studied in [19], [20], where closed-loop convergence is guaranteed based on the turnpike and controllability properties. For periodic operation, some research works of economic MPC are taken into account, see, e.g. [21], [22].

The main contribution of this paper is economic MPC for linear systems and convex cost functions with periodic operations in which the optimal periodic trajectory that minimizes the economic cost function is unknown. In hierarchical MPC schemes, this trajectory is computed off-line. In the proposed economic MPC design, instead of enforcing terminal state constraint over the prediction horizon, the economic cost function is optimized with a periodicity constraint at each time step considering all periodic trajectories in a period including the current state.

The proposed controller guarantees stability of the closedloop system, and under certain assumptions, convergence to the optimal periodic trajectory for the economic cost considered. To test convergence to the optimal trajectory an on-line test is provided based on the Karush-Khun-Tucker (KKT) conditions. Based on this analysis, we have proved that the closed-loop system may be trapped into a periodic trajectory different from the optimal steady one if the number of degrees of freedom is low and constraints are too tight. In this case, the closed-loop operational cost is decreasing until a constant value but more expensive than the planner cost.

Finally, we apply the proposed control strategy to a smart micro-grid to show its effectiveness. With the proposed economic MPC controller, the closed-loop trajectory can reach the planner one and the closed-loop operational cost converges to the planner cost. Besides, we also show a counter example that when the number of degrees of freedom is low, the closed-loop system state trajectory could be different from its planner trajectory.

The paper organization starts with problem statement in Section II. The economic MPC with periodic operations is formulated in Section III. The closed-loop properties are discussed in Section IV. Two case studies are shown in Section V. 


\section{Problem Statement}

Let us consider the following discrete-time linear system

$$
x_{k+1}=f\left(x_{k}, u_{k}\right) \text {, }
$$

where $x \in \mathbb{R}^{n_{x}}$ and $u \in \mathbb{R}^{n_{u}}$ denote the system state and the control input vectors, and $f(\cdot)$ is a linear function describing the system dynamics. We assume that the states $x_{k}$ and the control inputs $u_{k}$ of the system (1), $\forall k \in \mathbb{N}_{+}$are constrained by the convex sets $x_{k} \in \mathcal{X}_{k}$ and $u_{k} \in \mathcal{U}_{k}, \forall k \in \mathbb{N}$ with $\mathcal{X}_{k}=\mathcal{X}_{k+T}, \mathcal{U}_{k}=\mathcal{U}_{k+T}$ where $T$ is a period.

The system (1) is expected to be governed to achieve an economic benefit. The economic performance of the system (1) is measured by a time-varying economic stage cost function

$$
\ell_{k}\left(x_{k}, u_{k}, p_{k}\right)
$$

where $p_{k}$ is a time-varying exogenous signal (for example, the unit price of electricity) exhibiting a periodic behavior, that is $p_{k}=p_{k+T}$ corresponding to a period $T$. The main control objective is to minimize the closed-loop economic cost measured by $\ell_{k}\left(x_{k}, u_{k}, p_{k}\right)$. We consider that $\ell_{k}\left(x_{k}, u_{k}, p_{k}\right)$ is a strictly convex function, $\forall k \in \mathbb{N}_{+}$ and the periodicity of this economic stage cost function is given by $\ell_{k}\left(x_{k}, u_{k}, p_{k}\right)=\ell_{k+T}\left(x_{k+T}, u_{k+T}, p_{k+T}\right)$.

\section{ECONOMiC Model Predictive Control With PERIODIC OPERATIONS}

We now present the economic MPC design with periodic operations. As introduced above, we first propose an economic MPC planner to obtain the optimal periodic steady trajectory for the closed-loop analysis.

\section{A. Economic MPC Planner}

According to [10], [23], considering the periodicity, the infinite-horizon performance of the optimization problem (4) is equivalent to the following finite-horizon optimization problem:

$$
\underset{x_{0}, \ldots, x_{T}, u_{0}, \ldots, u_{T-1}}{\operatorname{minimize}} J_{T}(x, u)=\sum_{i=0}^{T-1} \ell_{i}\left(x_{i}, u_{i}, p_{i}\right),
$$

subject to

$$
\begin{aligned}
& x_{i+1}=f\left(x_{i}, u_{i}\right), \\
& x_{i} \in \mathcal{X}_{i}, \\
& u_{i} \in \mathcal{U}_{i}, \\
& x_{0}=x_{T} .
\end{aligned}
$$

Note that in the above formulation, we have chosen the time step 0 as the first step of one period. If a different step is chosen the functions would be different but would lead to an equivalent problem. Hence, we solve the optimization problem (3) once to obtain the optimal periodic steady planner trajectory.

\section{B. Economic MPC Formulation}

The economic cost function along a period $T$ is optimized based on a periodicity constraint. The economic MPC strategy can be implemented by solving the following optimization problem:

$$
\underset{x_{0}, \ldots, x_{T}, u_{0}, \ldots, u_{T-1}}{\operatorname{minimize}} J_{T}(x, u)
$$

subject to

$$
\begin{aligned}
& x_{i+1}=f\left(x_{i}, u_{i}\right), \\
& x_{i} \in \mathcal{X}_{i}, \\
& u_{i} \in \mathcal{U}_{i}, \\
& x_{0}=x_{T}, \\
& x_{j}=x_{k}, \quad j=\bmod (k, T) .
\end{aligned}
$$

Considering the periodic behavior, the optimization problem (4) is always initialized from the time instant 0 . At each sampling time $k$, this optimization problem is solved considering a fixed temporal window. Note that the current state $x_{k}$ is not always set as the first prediction state. Denote the optimal solution of the optimization problem (4) as $u^{*}$. Based on the receding horizon strategy, the optimal control action $u_{k}$ at time instant $k$ is

$$
u_{k}=u_{j}^{*}, j=\bmod (k, T) .
$$

\section{The Closed-LOOP PROPERTIES}

Considering that all the defined functions and constraints in the above optimization problems are convex, we reformulate the optimization problems (4) and (3) into a standard convex formulation.

Define

$$
z=\left[\begin{array}{llllll}
x_{0}{ }^{T} & \cdots & x_{T}^{T} & u_{0}^{T} & \cdots & u_{T-1}^{T}
\end{array}\right]^{T},
$$

and denote $J_{T}(x, u)$ as $J_{T}(z)$. We rewrite the optimization problem (3) in a standard convex form as follows

$$
\underset{z}{\operatorname{minimize}} J_{T}(z)
$$

subject to

$$
\begin{aligned}
& h_{r}(z) \leq 0, \quad r=1, \ldots, m \\
& g_{i}(z)=0, \quad i=1, \ldots, n
\end{aligned}
$$

where $h_{r}$ for $r=1, \ldots, m$ are convex functions and $g_{i}$ for $i=1, \ldots, n$ are affine/linear functions of system constraints $\mathcal{X}$ and $\mathcal{U}$. According to (6), we denote the optimal solution of the optimization problem (7) as $z^{p}$.

By convexity, it follows that there exist dual variables

$$
\lambda^{p}=\left[\begin{array}{c}
\lambda_{1}^{p} \\
\vdots \\
\lambda_{m}^{p}
\end{array}\right], \mu^{p}=\left[\begin{array}{c}
\mu_{1}^{p} \\
\vdots \\
\mu_{n}^{p}
\end{array}\right],
$$


such that we have the KKT necessary conditions of (7) as follows:

$$
\begin{aligned}
& \nabla J_{T}\left(z^{p}\right)+\sum_{r=1}^{m} \lambda_{r}^{p} \nabla h_{r}\left(z^{p}\right)+\sum_{i=1}^{n} \mu_{i}^{p} \nabla g_{i}\left(z^{p}\right)=0, \\
& h_{r}\left(z^{p}\right) \leq 0, \quad r=1, \ldots, m, \\
& g_{i}\left(z^{p}\right)=0, \quad i=1, \ldots, n, \\
& \lambda_{r}^{p} \geq 0, \quad r=1, \ldots, m, \\
& \lambda_{r}^{p} h_{r}\left(z^{p}\right)=0, \quad r=1, \ldots, m .
\end{aligned}
$$

Similarly, with $z$ in (6), the optimization problem (4) can be reformulated to a standard convex form as

$$
\underset{z}{\operatorname{minimize}} J_{T}(z)
$$

subject to

$$
\begin{aligned}
& h_{r}(z) \leq 0, \quad r=1, \ldots, m, \\
& g_{i}(z)=0, \quad i=1, \ldots, n, \\
& Q_{j} z=x_{k}, \quad j=\bmod (k, T),
\end{aligned}
$$

where the matrix $Q_{j}$ is chosen such that $x_{j}=x_{k}$ with $j=\bmod (k, T)$. We denote the optimal solution of the optimization problem (9) at time $k$ as $z_{k}$.

Therefore, there exist dual variables

$$
\lambda_{k}=\left[\begin{array}{c}
\lambda_{1, k} \\
\vdots \\
\lambda_{m, k}
\end{array}\right], \mu_{k}=\left[\begin{array}{c}
\mu_{1, k} \\
\vdots \\
\mu_{n, k}
\end{array}\right], \nu_{k}=\left[\begin{array}{c}
\nu_{1, k} \\
\vdots \\
\nu_{n_{x}, k}
\end{array}\right],
$$

such that we have the KKT necessary conditions of (9) as follows:

$$
\begin{aligned}
& \nabla J_{T}\left(z_{k}\right)+\sum_{r=1}^{m} \lambda_{r, k} \nabla h_{r}\left(z_{k}\right) \\
& \quad+\sum_{i=1}^{n} \mu_{i, k} \nabla g_{i}\left(z_{k}\right)+\sum_{l=1}^{n_{x}} \nu_{l, k} Q_{\bmod (k, T)}^{l}=0, \\
& h_{r}\left(z_{k}\right) \leq 0, \quad r=1, \ldots, m, \\
& g_{i}\left(z_{k}\right)=0, \quad i=1, \ldots, n, \\
& Q_{\bmod (k, T)} z_{k}=x_{\bmod (k, T)}, \\
& \lambda_{r} \geq 0, \quad r=1, \ldots, m, \\
& \lambda_{r} h_{r}\left(z_{k}\right)=0, \quad r=1, \ldots, m,
\end{aligned}
$$

where $Q_{\bmod (k, T)}^{l}$ denotes the transpose of the $l$-th row of $Q_{\bmod (k, T)}$.

We study the properties of the proposed controller in the following theorem.

Theorem 1: The closed-loop control system of (1) with (4) is stable. If the dual variables $\nu_{k}, \forall k \geq M$ corresponding to the equality constraint (10d) are zero after the time step $M$, then the closed-loop control system (1) with (4) has reached the planner trajectories $z^{p}$.

Proof: If the optimization problem (4) is feasible at time instant $k$, then it is also feasible at time instant $k+1$. To this end, we prove that $z_{k}$, the optimal solution at time instant $k$, is a feasible solution for the optimization problem (4) at time step $k+1$. Taking into account that the optimization variable defines a periodic trajectory, constraints (4b)-(4e) are satisfied by definition. Constraint (4f) is defined as follows:

$$
x_{j}=x_{k+1}, \quad j=\bmod (k+1, T),
$$

which is equivalent to

$$
x_{j+1}=x_{k+1}, \quad j=\bmod (k, T) .
$$

Taking into account that

$$
\begin{aligned}
x_{j+1} & =f\left(x_{j}, u_{j}\right), \\
x_{j} & =x_{k}, \quad j=\bmod (k, T), \\
u_{j} & =u_{k}, \quad j=\bmod (k, T),
\end{aligned}
$$

hold, the equality (11) follows.

Then, we prove that under certain assumptions, the closedloop system converges to the planner. By optimality [24], for the optimization problem (9), we have $J_{T}\left(z_{k+1}\right) \leq J_{T}\left(z_{k}\right)$, which implies the cost of the optimization problem (4) is a non-increasing sequence. Taking into account that by assumption $J_{T}(\cdot)$ is a strictly convex function, it is not possible that there exist two consecutive time instants $k$ and $k+1$ such that the costs $J_{T}\left(z_{k}\right)=J_{T}\left(z_{k+1}\right)$ with $z_{k} \neq z_{k+1}$. Hence, if $J_{T}\left(z_{k+1}\right)=J_{T}\left(z_{k}\right), \forall k \geq M$, the system (1) in closed-loop reaches a periodic steady trajectory, that is $z_{M}=$ $z_{M+1}=\cdots$.

Without loss of generality, we assume that $\bmod (M, T)=$ 0 . Then, a suboptimal solution $z^{s}$ can be taken from this periodic trajectory to be

$$
z^{s}=\left[\begin{array}{llllll}
x_{M}^{T} & \cdots & x_{M+T^{T}} & u_{M}^{T} & \cdots & u_{M+T-1}{ }^{T}
\end{array}\right]^{T} .
$$

The solution $z^{s}$ is also feasible for the optimization problem (3). On one hand, the closed-loop solution $z_{k}$ should be equal to the optimal solution $z^{s}$, that is $z^{s}=z_{k}$, $\forall k \geq M$. On the other hand, $z^{s}$ is an optimal solution of the optimization problem (4) such that $z^{s}, \forall k \geq M$ satisfies the KKT necessary conditions in (10). If the dual variables in $\nu_{k}$ are zero, then (10d) can be disabled. Therefore, $z^{s}$, $\forall k \geq M$ also satisfies the KKT necessary conditions in (8). Hence, we have

$$
z^{s}=z^{p}
$$

that is the closed-loop trajectory $z_{k}$ converges to the planner trajectory $z^{p}, \forall k \geq M$.

\section{CASe Studies}

\section{A. Economic Optimization of a Smart Micro-Grid}

We consider the control of a smart micro-grid that includes a battery bank, a DC bus, several energy sources (photovoltaic, wind, hydroelectric and diesel generators), and three electricity demands. In this system, the prices to supply electricity are different from different sources and the supplying availability of photovoltaic and wind generators is periodically time-varying depending on the environmental factors. 
Based on a flow model [25], the control-oriented model of this smart micro-grid is expressed in discrete-time as

$$
\begin{aligned}
x_{k+1} & =A x_{k}+B u_{k}+B_{p} d_{k}, \\
0 & =E_{u} u_{k}+E_{d} d_{k},
\end{aligned}
$$

where $x$ denotes the state of charge of the battery as the state variable, $u$ denotes the power flow through active links as input variable, and $d$ denotes the power demand as an exogenous input variable with $d_{k}=d_{k+T}, \forall k \in \mathbb{N}$. Based on a daily pattern, the period is considered as $T=24$ with the sampling time of one hour. System matrices in (13) are

$$
\begin{aligned}
& A=1, B=\left[\begin{array}{llllll}
0.95 & -1 & 0 & 0 & 0 & 0
\end{array}\right], \\
& E_{u}=\left[\begin{array}{lllllllll}
-1 & 1 & 1 & 1 & 1 & 1 & -1 & -1 & -1
\end{array}\right] \text {, } \\
& E_{d}=\left[\begin{array}{lll}
-1 & -1 & -1
\end{array}\right],
\end{aligned}
$$

and considering that there is no direct connection between the battery and power demands, $B_{p}$ is a zero matrix of appropriate dimension.

The economic stage cost function (2) for controlling this grid is defined as [6]

$$
\ell_{k}\left(x_{k}, u_{k}, p\right)=\alpha_{1} p^{T} u_{k}+\alpha_{2}\left\|\xi_{k}\right\|_{2}^{2}+\alpha_{3}\left\|x_{k}\right\|_{1}^{2},
$$

where $\alpha_{1}, \alpha_{2}$ and $\alpha_{3}$ are prioritization weights, $\|\cdot\|_{1}$ and $\|\cdot\|_{2}$ denote the 1-norm and the 2-norm of a vector, $p$ denotes a vector of prices for active links and the term $p^{T} u_{k}$ directly measures the economic operational costs associated with power transmissions. Since the signal $p$ is constant, it also satisfies $p_{k}=p_{k+T}$. The slack variable $\xi_{k}$ is used to consider a soft constraint on the minimum state of charge

$$
x_{k} \geq x_{s}-\xi_{k},
$$

where $x_{s}$ denotes the safety level to keep a certain amount of energy in the battery bank to deal with the underlying power demands guaranteeing the energy supply. Additionally, the term $\left\|x_{k}\right\|_{1}^{2}$ penalizes the level of the battery bank to keep the minimal required energy forcing the convergence to the periodic steady state behaviour.

The constraint on the state $x_{k}$ (state of charge), $\forall k \in \mathbb{N}$ is set as

$$
x_{k} \in \mathcal{X}, \forall k \in \mathbb{N}, \mathcal{X}=\{x \in \mathbb{R} \mid 0 \leq x \leq 100\},
$$

and the constraint on the input $u_{k}, \forall k \in \mathbb{N}$ is set to be a time-varying set $u_{k} \in \mathcal{U}_{k}$ with $\mathcal{U}_{k}=\mathcal{U}_{k+T}$ considering that the daily periodicity of photovoltaic and wind generators.

The proposed EMPC approach has been tested using a simulation that has been carried out for 5 days (120 hours). $\alpha_{1}, \alpha_{2}$ and $\alpha_{3}$ are chosen as $10,0.1$ and 0.01 , respectively, to obtain the desired trade-off among the different objectives. The safety level is set as $x_{s}=35 \%$. Note that all the variables in this model are expressed using international units. The considered power demands $d_{k}=d_{k+T}$ with $T=24$ is given as shown in Fig. 1. This demand is assumed available to the MPC in the prediction horizon. We solve the optimization problem (4) and (3) by means of the YALMIP toolbox [26] and MOSEK solver [27].

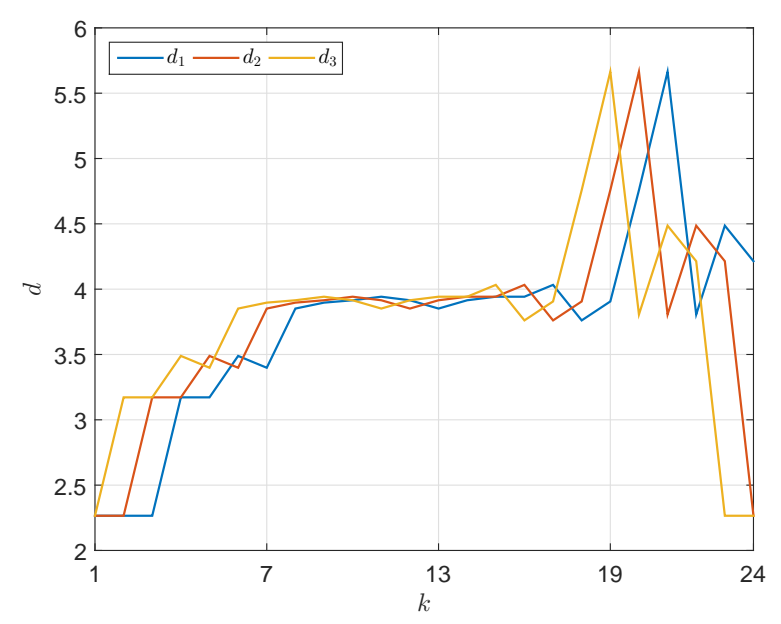

Fig. 1. The power demand data of the smart micro-grid.

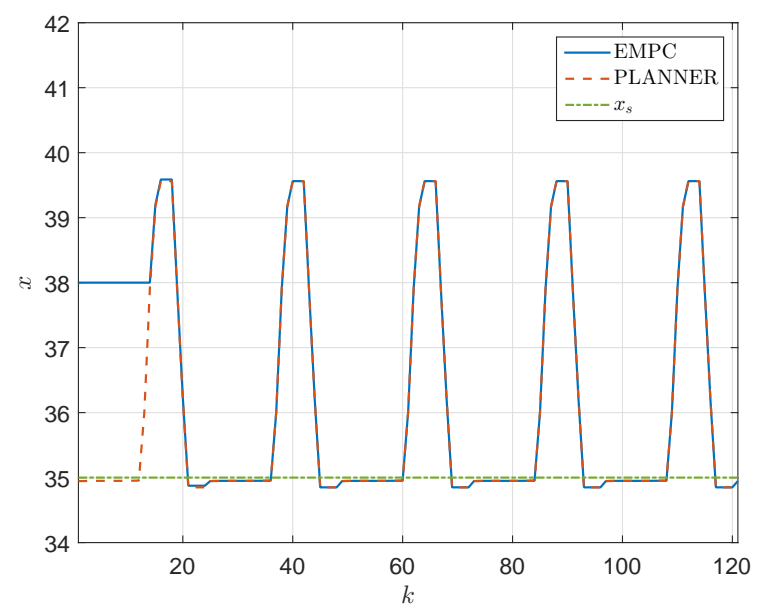

Fig. 2. The closed-loop system state trajectory of the smart micro-grid.

The closed-loop system state and control input trajectories are shown in Fig. 2 and 3. From both figures, the closedloop trajectories converge to the planner ones after 14 time steps. In Fig. 3, the optimal inputs are obtained within limits and the periodically time-varying constraint is also satisfied as shown in Fig. 3(b). Since the solar energy has a lower cost, this optimal input reaches its maximum to achieve the optimum performance from the economic point of view.

The closed-loop operational cost is computed using the defined economic cost function (14) and the optimal closedloop solutions. The cost result is shown in Fig. 4. As the closed-loop trajectory is approaching the planner, the cost is also converging to the planner cost which is optimal. Besides, with the economic MPC controller in (4), we also extract dual variables from the solver in order to check Theorem 1 to certify that the steady state solution has been reached. As shown in Fig. 5, the dual variables $\nu_{k}$ corresponding to the equality constraint (10d) are zero after time step $M=14$ and the closed-loop trajectory reaches the planner. 


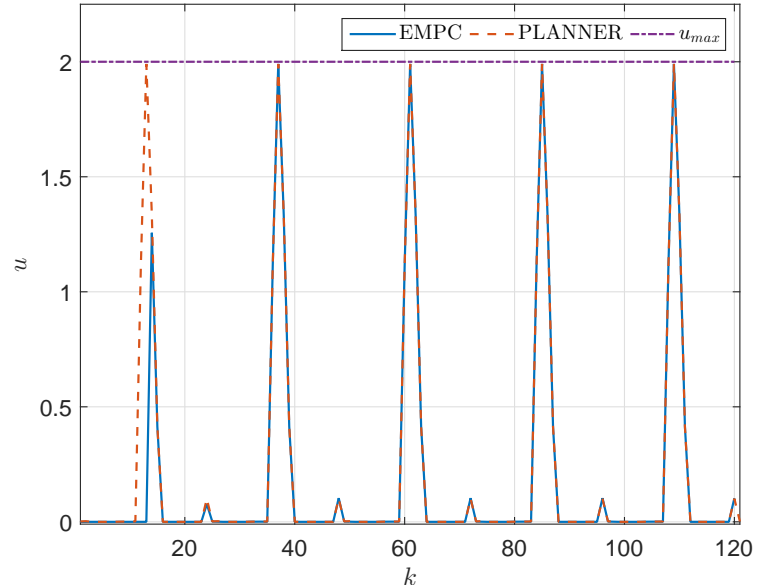

(a) Battery $u_{1}$

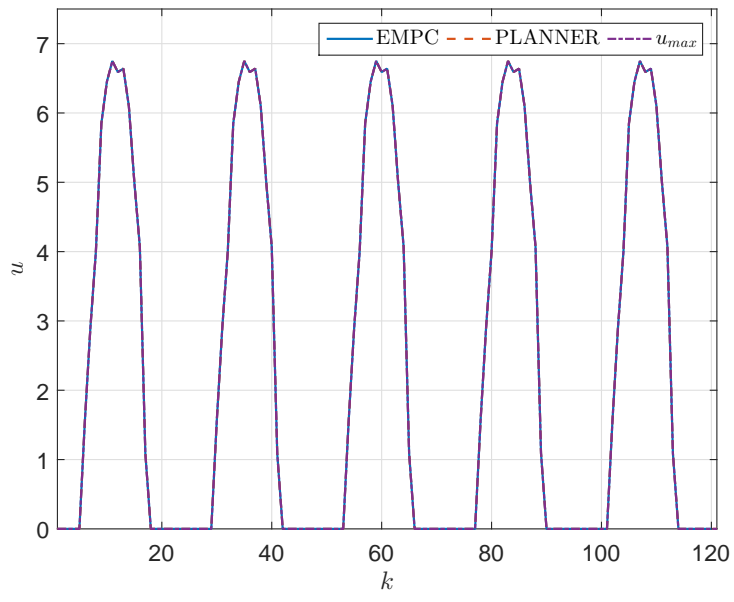

(b) Photovaltaic generator $u_{3}$.

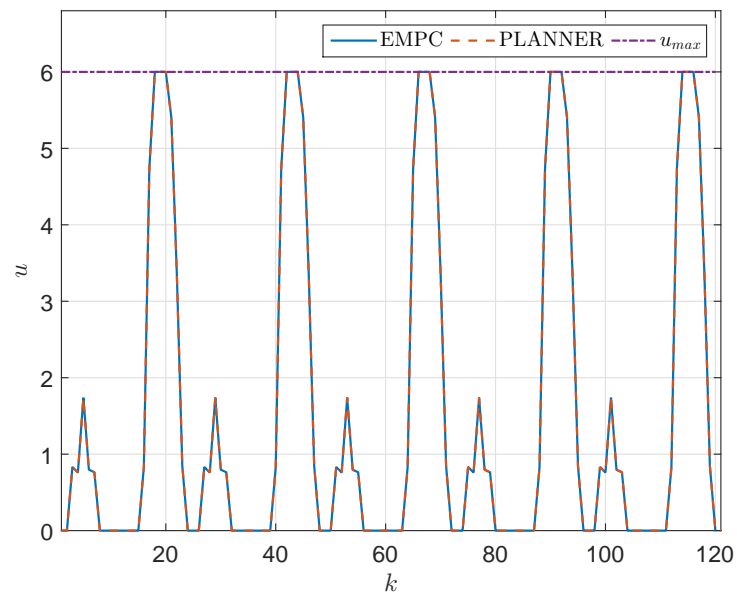

(c) Diesel generator $u_{6}$

Fig. 3. The closed-loop control input trajectories of the smart micro-grid.

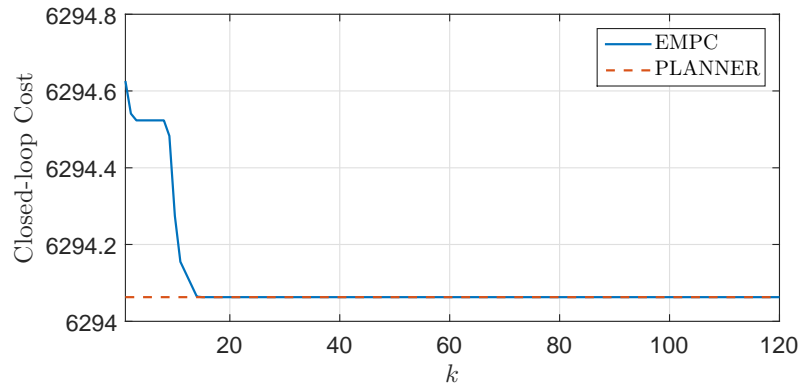

Fig. 4. The closed-loop operational cost of the smart micro-grid.

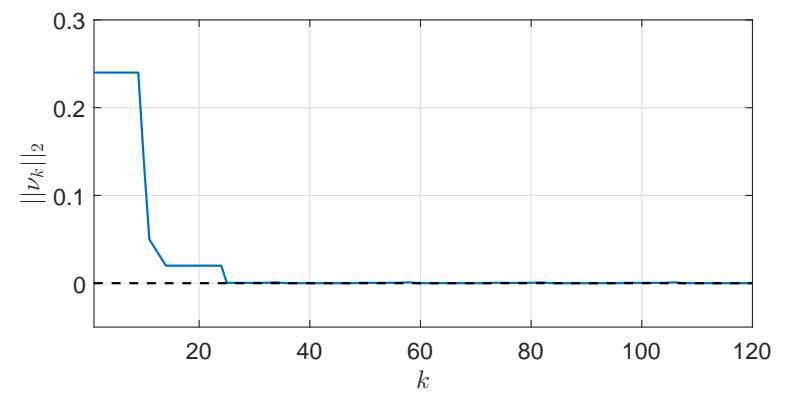

Fig. 5. The measure of dual variables $\nu_{k}$ of the smart micro-grid.

\section{B. A Counter Example}

As second example is presented where the closed-loop system reaches a periodic trajectory different from the planner because the constraints are very stringent. Given the discretetime system in the form of (13a) with

$$
A=\left[\begin{array}{cc}
0.5 & 0.5 \\
1 & 0.25
\end{array}\right], B=\left[\begin{array}{l}
1 \\
1
\end{array}\right], B_{p}=\left[\begin{array}{l}
1 \\
0
\end{array}\right],
$$

where $d$ is a periodic known input signal. This system is controlled by the proposed economic MPC in (4) with the period of $T=3$. The corresponding optimization problem is given by the formulation in (9) with $m=n=1, J_{T}(z)=$ $\frac{1}{2} z^{T} H z+f^{T} z$, and

$$
\begin{aligned}
H & =\operatorname{diag}\left(\left[\begin{array}{lllllllll}
1 & 1 & 10 & 1 & 1 & 20 & 1 & 1 & 10
\end{array}\right]\right), \\
f & =\left[\begin{array}{lllllllll}
0.1 & 0.1 & 0.1 & 0.1 & 0.1 & 0.1 & 0.1 & 0.1 & 0.1
\end{array}\right],
\end{aligned}
$$

where $\operatorname{diag}(\cdot)$ denotes a diagonal matrix with diagonal elements. We also solve the planner (7) with the same initial conditions using linear programming in MATLAB.

The closed-loop system state trajectories in 120 steps are shown in Fig. 6. In Fig. 6, considering that the period of this counter example is $T=3$, the closed-loop trajectories of $x_{1}$ and $x_{2}$ arrive at periodic trajectories but are different from the planner trajectories. From Fig. 7, the closed-loop operational cost is always decreasing to arrive at a constant when the closed-loop trajectories reach periodic trajectories. But since the periodic trajectories are different than the planner ones, the closed-loop operational cost is more expensive than the planner cost as shown in Fig. 7.

Besides, the 2-norm of the dual variable $\nu_{k}$ is plotted in Fig. 8, where we can see that the elements of this dual 


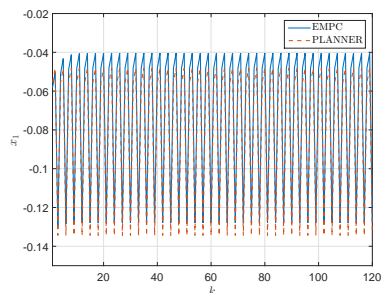

(a) $x_{1}$

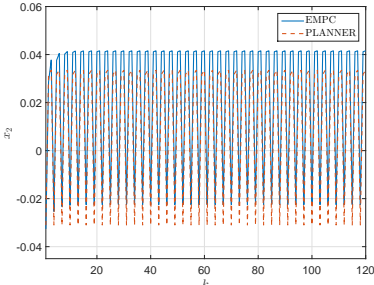

(b) $x_{2}$
Fig. 6. The closed-loop system state trajectories of the counter example.

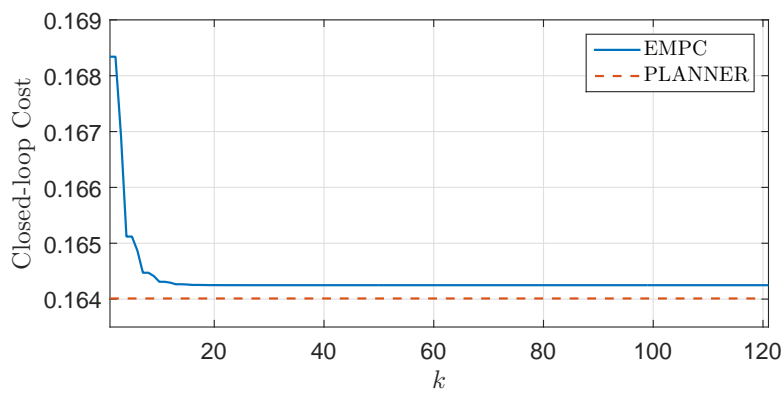

Fig. 7. The closed-loop operational cost of the counter example.

variables are not zero at any time since the closed-loop trajectories cannot reach the planner.

\section{ACKNOWLEDGEMENT}

This work has been partially funded by the Spanish State Research Agency (AEI) and the European Regional Development Fund (ERFD) through the projects ECOCIS (ref. DPI2013-48243-C2) and DEOCS (ref. DPI2016-76493C3), and the María de Maeztu Seal of Excellence to IRI (MDM-2016-0656).

\section{REFERENCES}

[1] D. Mayne, "Model predictive control: Recent developments and future promise," Automatica, vol. 50, no. 12, pp. 2967-2986, 2014.

[2] M. Ellis, J. Liu, and P. Christofides, Economic Model Predictive Control: Theory, Formulations and Chemical Process Applications. Springer, 2017.

[3] Y. Wang, V. Puig, and G. Cembrano, "Non-linear economic model predictive control of water distribution networks," Journal of Process Control, vol. 56, pp. 23-34, 2017.

[4] Y. Wang, G. Cembrano, V. Puig, M. Urrea, J. Romera, D. Saporta, and J. Valero, "Model predictive control of water networks considering flow and pressure," in Real-time Monitoring and Operational Control of Drinking-Water Systems. Springer, 2017, pp. 251-267.

[5] J. Zeng and J. Liu, "Economic model predictive control of wastewater treatment processes," Industrial \& Engineering Chemistry Research, vol. 54, no. 21, pp. 5710-5721, 2015.

[6] M. Pereira, D. Limon, D. Muñoz de la Peña, L. Valverde, and T. Alamo, "Periodic economic control of a nonisolated microgrid," IEEE Transactions on Industrial Electronics, vol. 62, no. 8, pp. 52475255, 2015.

[7] M. Pereira, D. Limon, D. Muñoz de la Peña, and D. Limon, "Robust economic model predictive control of a community micro-grid," Renewable Energy, vol. 100, pp. 3-17, 2017.

[8] S. Liu, J. Zhang, and J. Liu, "Economic MPC with terminal cost and application to an oilsand primary separation vessel," Chemical Engineering Science, vol. 136, pp. 27-37, 2015.

[9] O. Santander, A. Elkamel, and H. Budman, "Economic model predictive control of chemical processes with parameter uncertainty," Computers \& Chemical Engineering, vol. 95, pp. 10-20, 2016.

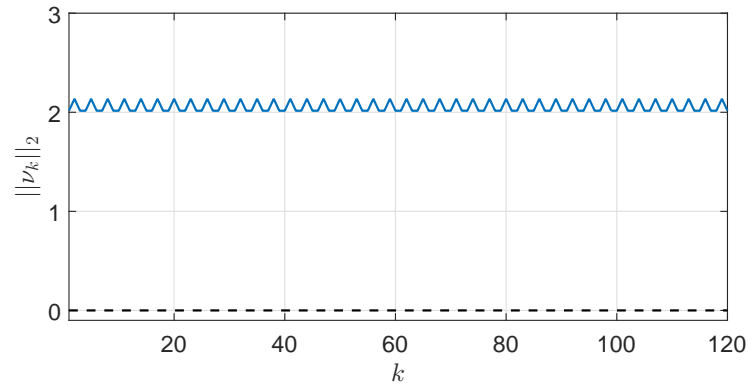

Fig. 8. The measure of dual variables $\nu_{k}$ of the counter example.

[10] D. Limon, M. Pereira, D. Muñoz de la Peña, T. Alamo, and J. Grosso, "Single-layer economic model predictive control for periodic operation,” Journal of Process Control, vol. 24, no. 8, pp. 1207-1224, 2014.

[11] Y. Wang, T. Alamo, V. Puig, and G. Cembrano, "Periodic economic model predictive control with nonlinear-constraint relaxation for water distribution networks," in IEEE Conference on Control Applications (CCA), Buenos Aires, Argentina, 2016, pp. 1167-1172.

[12] Y. Wang, V. Puig, and G. Cembrano, "Economic MPC with periodic terminal constraints of nonlinear differential-algebraic-equation systems: Application to drinking water networks," in European Control Conference, Aalborg, Denmark, 2016, pp. 1013-1018.

[13] Y. Wang, J. Ramón Salvador, D. Muñoz de la Peña, V. Puig, and G. Cembrano, "Periodic nonlinear economic model predictive control with changing horizon for water distribution networks," in 20th IFAC World Congress, Toulouse, France, 2017.

[14] M. Ellis, H. Durand, and P. Christofides, "A tutorial review of economic model predictive control methods," Journal of Process Control, vol. 24 , no. 8 , pp. $1156-1178,2014$.

[15] R. Amrit, J. Rawlings, and D. Angeli, "Economic optimization using model predictive control with a terminal cost," Annual Reviews in Control, vol. 35, no. 2, pp. 178-186, 2011.

[16] D. Angeli, R. Amrit, and J. Rawlings, "On average performance and stability of economic model predictive control," IEEE Transactions on Automatic Control, vol. 57, no. 7, pp. 1615-1626, 2012.

[17] M. Müller, D. Angeli, and F. Allgöwer, "On the performance of economic model predictive control with self-tuning terminal cost," Journal of Process Control, vol. 24, no. 8, pp. 1179-1186, 2014.

[18] S. Liu and J. Liu, "Economic model predictive control with extended horizon," Automatica, vol. 73, pp. 180-192, 2016.

[19] L. Grüne, "Economic receding horizon control without terminal constraints," Automatica, vol. 49, no. 3, pp. 725-734, 2013.

[20] L. Grüne and M. Stieler, "Asymptotic stability and transient optimality of economic MPC without terminal conditions," Journal of Process Control, vol. 24, no. 8, pp. 1187-1196, 2014.

[21] B. Houska, "Enforcing asymptotic orbital stability of economic model predictive control," Automatica, vol. 57, pp. 45-50, 2015.

[22] M. Müller and L. Grüne, "Economic model predictive control without terminal constraints for optimal periodic behavior," Automatica, vol. 70, pp. 128-139, 2016.

[23] D. Limon, M. Pereira, D. Muñoz de la Peña, T. Alamo, C. Jones, and M. Zeilinger, "MPC for tracking periodic references," IEEE Transactions on Automatic Control, vol. 61, no. 4, pp. 1123-1128, 2016.

[24] S. Boyd and L. Vandenberghe, Convex optimization. Cambridge University Press, 2004.

[25] C. Ocampo-Martinez, V. Puig, G. Cembrano, and J. Quevedo, "Application of MPC strategies to the management of complex networks of the urban water cycle," IEEE Control Systems, vol. 33, no. 1, pp. $15-41,2013$

[26] J. Löfberg, YALMIP: A Toolbox for Modeling and Optimization in MATLAB, 2004. [Online]. Available: http://users.isy.liu.se/johanl/yalmip

[27] MOSEK ApS, The MOSEK optimization toolbox for MATLAB manual. Version 7.1 (Revision 28)., 2015. [Online]. Available: http://docs.mosek.com/7.1/toolbox/index.html 